\title{
CHIRAL CHROMATOGRAPHIC ANALYSIS AND ABSOLUTE STEREOCHEMISTRY OF RACEMIC CHLORPHENERAMINE USING CHIRAL HPLC-CD-OR AND TD-DFT CALCULATIONS
}

\author{
MARCELO A. MUÑOZ \\ Instituto de Ciencias Químicas, Facultad de Ciencias, Universidad Austral de Chile, Casilla 567, Valdivia, Chile.
}

\section{ABSTRACT}

A chiral HPLC analysis combined with time-dependent density functional theory (TD-DFT) calculations was used for the assignment of the absolute configuration (AC) of both enantiomers of the antihistamine drug chlorpheniramine. Circular dichroism and optical rotation detections coupled to the chromatographic system (HPLC-CD-OR) permitted the on-line measurement of the CD spectra and $[\alpha]_{670}$ values, while TD-DFT at the B3LYP/TZVP//B3LYP/VDZ(P) and B3LYP/ aug-cc-pVDZ//B3LYP/VDZ(P) levels of theory for CD and OR, respectively, delivered theoretical chiroptical properties in the gas and solution states. Both calculations were consistent in predicting the correct combination of CD spectra and $[\alpha]_{670}$ sign and therefore permitted the absolute configuration (AC) assignment of the optical isomers. The AC proposed using this methodology agreed with a previous X-ray study.

Keywords: HPLC-CD-OR, circular dichroism detection, optical rotation detection, DFT calculations.

\section{INTRODUCTION}

Chiral organic compounds are commonly encountered when studying secondary metabolites and biologically active substances, and therefore the assessment of their absolute configuration (AC) is highly desirable. ${ }^{1}$

Nevertheless, the success of studies aiming to establish the AC of optically active substances, using methodologies such as chemical correlation, single-crystal X-ray diffraction, ${ }^{2}$ or electronic/vibrational circular dichroism spectroscopies $^{3}$, are highly dependent on the amount and purity of the samples used. In the other hand, the measurement of chiroptical properties through chromatographic techniques, enable the use of smaller and more complex samples. Therefore, dedicated instruments based on electronic circular dichroism (HPLC-CD) and optical rotation (HPLC-OR), have become commercially available, and found a variety of uses in the different fields of chemistry. ${ }^{4}$

Additionally to the chiroptical measurements, in most cases, the $\mathrm{AC}$ of a given compound can only be reached through comparison of experimental and calculated data, the later obtained through computational chemistry. While advances in the calculation of chiroptical properties have permitted the AC determination of many compounds, the use of a single chiroptical technique for these studies involve a significant risk of wrong assignments, and therefore the simultaneous use of at least two of these methods is strongly recommended to assign to a more reliable AC. ${ }^{5,6}$ In this context, an HPLC-CD-OR analysis followed by quantum-chemical calculations allows the use of two chiroptical properties simultaneously, and therefore leads to a more confident AC assignment of the studied substance. Additionally, when the method is applied to racemic mixtures, the OR measurement not only adds confidence to the initial assignment, but also relates a particular isomer with an OR sign, which is a more accessible chiroptical property to be measured.

We recently reported the use of this approach in the study of the chiroptical properties of racemic trans-stilbene oxide ${ }^{7}$ and the absolute configuration assignment of the tropane alkaloid 6-benzoyl-3-tropanol, ${ }^{8}$ both in agreement with literature data and VCD observations, respectively.

In this work, we further the use of chiral HPLC-CD-OR analysis and time-dependent density functional theory (TD-DFT) calculations for the AC assignment of racemic mixtures by applying it to racemic chlorpheniramine (1) (Fig. 1). Chlorpheniramine is a well-known antihistamine used to alleviate the symptoms of allergic conditions and is commonly marketed as the maleate of the racemic mixture. ${ }^{9}$ It has a single asymmetric tertiary carbon bearing a dimethylethylamine aliphatic substituent, along with an o-pyridine and a p-chlorophenyl aromatic rings. And although its absolute configuration has been established in the past, ${ }^{10}$ the present application will allow a better understanding of the potential and limitations of the proposed methodology, through applying it to a substance of larger conformational flexibility.

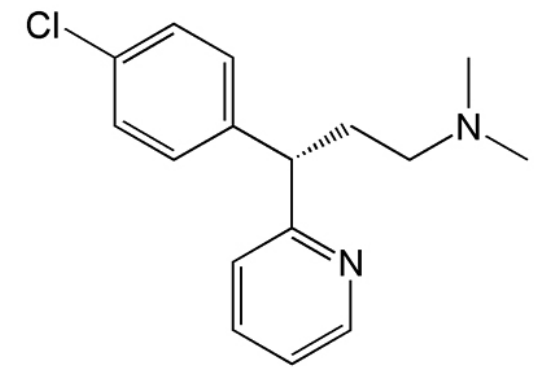

Fig. 1. Structure of chlorpheniramine (1). Only one enantiomer is shown.

2.

\section{MATERIALS AND METHODS}

2.1 Chemicals

$( \pm)$-Chlorpheniramine maleate $(99 \%)$ was acquired from Sigma (SigmaAldrich Química Ltda., Chile). HPLC-grade solvents n-hexane and 2-propanol were acquired from Merck (Merck S.A., Chile). All chemicals and solvents were used directly without further purification.

2.2 Chromatographic Conditions

Chromatographic analyses were performed on a Shimadzu HPLC chromatograph equipped with LC-20AT and LC-10ADvp pumps, a SIL10 Avp autosampler, and a CTO-10ACvp column oven. Coupled in series to this array, a JASCO CD-2095plus UV-CD detector (25-mm path length and 44- $\mu \mathrm{L}$ internal volume flow cell) was used for CD measurements, while a PDRChemical LLR advanced laser polarimeter detector (670-nm diode laser, 25$\mathrm{mm}$ path length and $18-\mu \mathrm{L}$ internal volume flow cell), was used in-line for OR measurements. UV, CD and OR signals from both detectors were acquired with JASCO ChromPass software version 1.7.403.1.

Chiral separations were then performed at $25.0{ }^{\circ} \mathrm{C}$ on a ChiralPak AD-H column ( $250 \mathrm{~mm}, 4.5 \mathrm{~mm}, 5 \mu \mathrm{m}$, Daicel Inc.) with a mobile phase composed by a mixture of n-hexane and 2-propanol 9:1.

UV and CD spectra were acquired simultaneously for both enantiomers in each racemic mixture using the stop-flow technique ${ }^{11}$ and following the same procedures described in the stereochemical study of trans-stilbene oxide. ${ }^{7}$ Solutions of 1 with appropriate concentrations (between 1 and $10 \mu \mathrm{g} / \mu \mathrm{L}$ ) were prepared by dissolving in the corresponding mobile phase in $5-\mathrm{mL}$ volumetric flasks, and samples of these solutions were filtered and analysed using 1- to $20-\mu \mathrm{L}$ injections

$[\alpha]_{670}$ values were extracted by measuring the height and half-height width of each OR peak and then applying the Gaussian peak model. ${ }^{12}$

2.3 Computational Calculations

All conformations found in the first $10 \mathrm{kcal} / \mathrm{mol}$ energy window of a molecular mechanics force field (MMFF) conformational search, were submitted to geometry optimization and vibrational calculations at the B3LYP/ VDZ(P) level of theory. The same procedure was performed separately using the conductor-like screening model (COSMO) $)^{13}$ with a dielectric constant equivalent to that of the mobile phase. The corresponding Boltzmann 
distributions produced relative abundances for each conformation, and only those found to be relevant were considered for $\mathrm{CD}$ and $\mathrm{OR}$ calculations. Calculated CD spectra for each individual conformation were obtained as described previously for trans-stilbene oxide ${ }^{7}$ at the B3LYP/TZVP level of theory, and then plotted as Gaussian bands with half-widths of $15 \mathrm{~nm}$ using the SpecDis software package. ${ }^{14}$ In the same way, $[\alpha]_{670}$ values were obtained from calculations of the average optical rotation tensor $\beta$ at the B3LYP/augcc-pVDZ level of theory. Finally, single weighted CD spectra and $[\alpha]_{670}$ values were obtained by considering the relative abundances of the corresponding Boltzmann distribution. Conformational searches were performed using the Spartan'14 software (Wavefunction Inc, Irvine, CA 92612, USA). Geometry optimizations and free energy calculations were carried out using the ORCA software package, ${ }^{15}$ while TD-DFT calculations were carried out using the NWChem software package. ${ }^{16}$

\section{RESULTS AND DISCUSSION}

Chromatographic conditions for the enantiomeric resolution of chlorpheniramine (1) can be found in commercial catalogues of chiral HPLC columns, ${ }^{17}$ and under these normal phase conditions, experimental $\mathrm{CD}$ and OR data (Fig. 2 and Table 1) were obtained on-line from HPLC runs, revealing that the (+)-1 and (-)-1 isomers eluted first $(6.5 \mathrm{~min})$ and second $(7.2 \mathrm{~min})$, respectively.

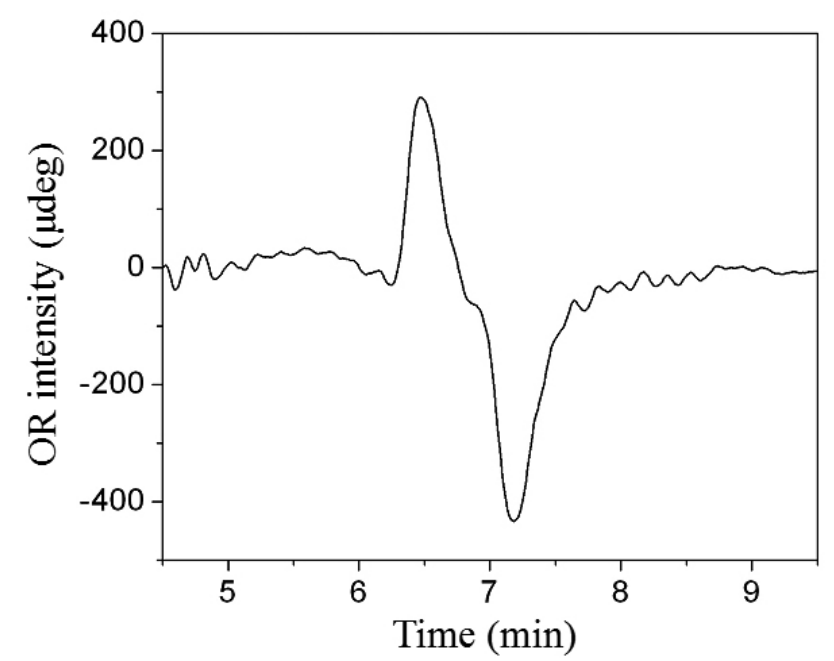

Fig. 2. OR chromatogram of the enantioselective separation of ( \pm -1: detection at $670 \mathrm{~nm}$.

On the other hand, 39 low-energy conformations were found in the conformational search for $\mathbf{1}$, and therefore additional steps were needed to avoid the large computational cost of considering all of the conformers in the chiroptical properties calculations. Ultimately, only six conformations accounted for $95.6 \%$ of the free energy Boltzmann distribution, and therefore only these conformations were considered further (Fig. 3).

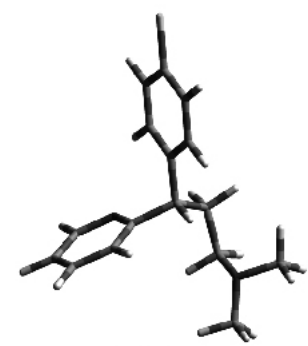

(S)-1a

$\mathbf{G}=-1189.32491289 \mathrm{Eh}$

$51.9 \%$

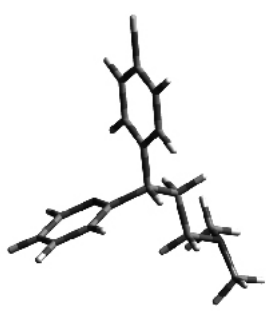

(S)-1d

$\mathbf{G}=-1189.32227016 \mathrm{Eh}$

$3.2 \%$

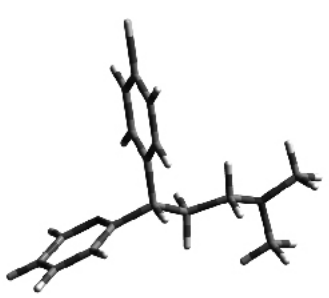

(S) $-1 \mathbf{b}$

$\mathbf{G}=-1189.32444235 \mathrm{Eh}$

$31.5 \%$

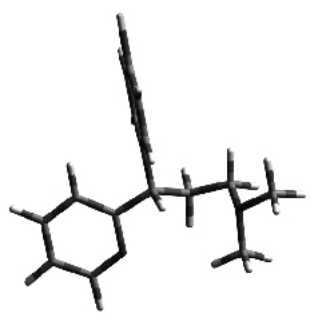

(S)-1e

$\mathbf{G}=-1189.32199230 \mathrm{Eh}$

$2.4 \%$

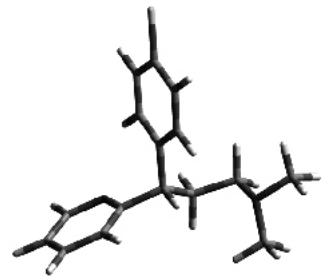

(S)-1c

$\mathbf{G}=-1189.32322938 \mathrm{Eh}$

$8.7 \%$

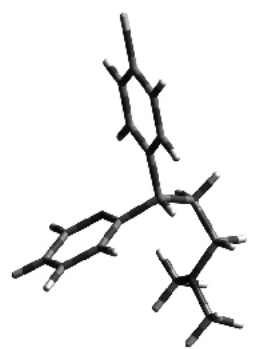

(S)-1f

$\mathbf{G}=-1189.32195941 \mathrm{Eh}$

$2.3 \%$

Fig. 3. Geometry-optimized conformations, Gibbs free enthalpies $(\mathrm{G})$ and relative abundances of $(+)-(S)-\mathbf{1}$ at the B3LYP/VDZ(P) level of theory. 
Consequently, comparison of experimental and calculated CD spectra obtained through these approximations clearly suggest that the first and second eluted peaks correspond to $(+)-(S)-\mathbf{1}$ and $(-)-(R)-\mathbf{1}$, respectively, as shown in Fig. 4. The calculated OR data also pointed to the same assignment by predicting the $(R)$ and $(S)$ isomers to be levo- and dextrorotatory, respectively, (Table 1) which also agrees with the reported AC of $\mathbf{1}$ from a previous X-ray study. ${ }^{10}$

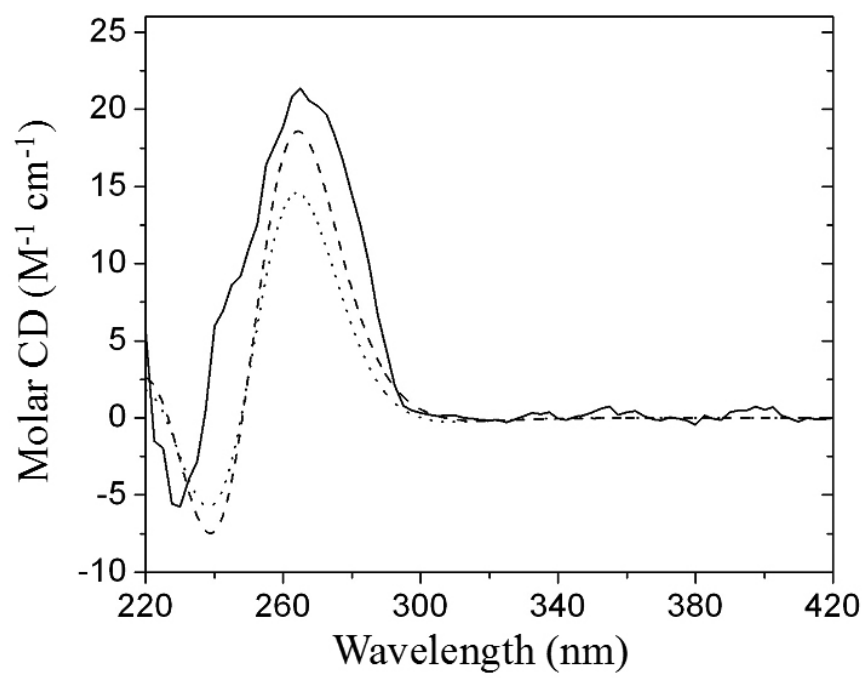

Fig. 4. Experimental (continuous), calculated in the gas phase (dashed) and calculated in solution (dotted) CD spectra of $(+)-(S)-1$ (flow cell concentration of $\left.1.6 \times 10^{-5} \mathrm{M}\right)$. The CD spectrum was calculated at the B3LYP/TZVP//B3LYP/VDZ(P) level of theory and shifted $5 \mathrm{~nm}$ towards shorter wavelengths for better comparison with experimental data.

Table 1 Experimental and calculated specific optical rotations ${ }^{\mathrm{a}}[\alpha]_{670}$ for chlorpheniramine (1).

\begin{tabular}{|c|c|c|c|c|c|c|c|c|}
\hline & \multicolumn{8}{|c|}{$(S)-1$} \\
\hline & $\mathbf{a}$ & b & c & d & e & f & g & $\mathbf{h}$ \\
\hline Conf $_{\text {gas }}{ }^{b}$ & +864.4 & +150.1 & +208.1 & +489.6 & -295.5 & +292.8 & - & - \\
\hline Conf $_{\text {sol }} \mathrm{c}$ & +61.6 & +51.8 & +313.6 & +139.4 & -55.5 & +75.1 & -264.2 & +1729.0 \\
\hline $\operatorname{Avg}_{\text {gas }}{ }^{\mathrm{d}}$ & \multicolumn{8}{|c|}{+529.6} \\
\hline $\operatorname{Avg}_{\text {sol }}{ }^{d}$ & \multicolumn{8}{|c|}{+146.6} \\
\hline $\mathrm{Expt}^{\mathrm{e}}$ & \multicolumn{8}{|c|}{$+33.8^{\mathrm{g}}$} \\
\hline
\end{tabular}

${ }^{a}$ Specific rotations in degrees $\left[\mathrm{dm} / \mathrm{g} / \mathrm{cm}^{3}\right]^{-1} \cdot{ }^{\mathrm{b}}$ Calculated in the gas phase at the B3LYP/aug-cc-pVDZ//B3LYP/VDZ(P) level of theory. ${ }^{\mathrm{c}}$ Calculated in solution state at the B3LYP/aug-cc-pVDZ//B3LYP/ VDZ(P) level of theory using COSMO. ${ }^{\mathrm{d}}$ Weighted average from the corresponding Boltzmann distribution. ${ }^{\mathrm{e}}$ Measured from chromatograms using the Gaussian peak method. ${ }^{\mathrm{g}}$ The measured $[\alpha]_{670}$ of the opposite isomer was -35.6 .

Nevertheless, TD-DFT calculations largely overestimated the OR of $\mathbf{1}$, with the theoretical $[\alpha]_{670}$ being almost 20 times higher than the measured value when solvent was not considered. However, solution state modelling in the conformational distribution of $\mathbf{1}$ significantly reduced this overestimation to less than 5 times the experimental value. A detailed inspection of the $[\alpha]$ values in Table 1 shows that this reduction is primarily due to the much lower values calculated for each conformation, particularly $\mathbf{1 a}, \mathbf{1 b}$, and 1d. More interestingly, the conformations obtained through COSMO remained very similar to the corresponding gas phase conformers but had noticeably different OR intensities.

The overestimation leads us to a closer inspection of the literature, which revealed that most applications of OR calculations refer to non-aromatic ${ }^{5,18,19}$ or fairly rigid $^{20,21,22,23}$ systems in which $[\alpha]$ do not primarily depend on chromophoric, free-rotating groups. From the few exceptions available, the cases of flavanone ${ }^{24}$ and (-)-naringenin ${ }^{25}$ suggest that these overestimations might be characteristic of such systems due to the high dependence of $[\alpha]$ on the rotation of aromatic groups. This behaviour is apparently not properly modelled by the lowest energy orientation alone. Specifically for $\mathbf{1}$, the conformational distribution in the gas phase revealed that 5 of the 6 low energy conformers $(\mathbf{1 a}, \mathbf{1 b}, \mathbf{1 c}, \mathbf{1 d}$ and 1f) share the same disposition as the two aromatic groups, while differing in the overall $N, N$-dimethylethylamine moiety conformation. A more detailed analysis of calculated OR data for each of these conformations (Table 1) showed that the same 5 conformers also share the same sign and high absolute values for this property, while the remaining disposition (1e) had a large $[\alpha]$ of opposite sign. This confirms the high dependency of OR on the relative orientation of aromatic groups in $\mathbf{1}$ and therefore suggests an equivalent explanation for the overestimations obtained herein.

\section{CONCLUSIONS}

The use of chiral HPLC with CD and OR detection (HPLC-CD-OR), along with TD-DFT computational calculations, is well suited to assign the absolute configuration of both enantiomers in racemic chlorpheniramine (1). The high molecular flexibility of compound 1 required the use of a Boltzmann distribution to define which conformations were relevant and restrained the property calculations to only these conformations. The calculated CD spectra compared well with the corresponding experimental measurements, but the theoretical $[\alpha]_{670}$ values were greatly overestimated. This appeared to be related to the presence of two free-rotating aromatic groups, for which OR intensities are highly dependent. Both chiroptical properties suggested that the AC of $\mathbf{1}$ was $(-)-(R)-1$ and $(+)-(S)-\mathbf{1}$, in agreement with a previous X-ray study.

\section{ACKNOWLEDGEMENT}

The authors acknowledge financial support from Universidad Austral de Chile (project DID-UACH S2013-02) and CONICYT-Chile (project FONDECYT 1141071) 


\section{REFERENCES}

1. Lemke T.L.; Williams D.A. Foye's Principles of Medicinal Chemistry Philadelphia: Lippincott Williams \& Wilkins; 2008. 38-43.

2. H.D. Flack, G. Bernardinelli, Chirality 20, 681-690, (2008)

3. G.H. Wagnière, On the interaction of light with molecules: pathways to the theoretical interpretation of chiroptical phenomena, in: N. Berova, P.L. Polavarapu, K. Nakanishi, R.W. Woody (Eds.), Comprehensive Chiroptical Spectroscopy Vol. 1, John Wiley \& Sons, New York, 2012, pp. 3-34.

4. L. Kott, W.B. Holzheuer, M.M. Wong, G.K. Webster, J. Pharm Biomed. Anal. 43, 57-65, (2007)

5. P.J. Stephens, J.J. Pan, F.J. Devlin, K. Krohn, T. Kurta'n, J. Org Chem. 72, 3521-3526, (2007)

6. P.J. Stephens, D.M. McCann, F.J. Devlin, A.B. Smith III, J. Nat. Prod. 69, 1055-1064, (2006)

7. J.P. Castillo-González, N.I. González-Peña, M.A. Muñoz, J. Chil. Chem. Soc. 60, 2642-2644. (2016)

8. M.A. Muñoz, N. González, P. Joseph-Nathan, J. Sep. Sci. 39, 2720 2727. (2016)

9. British Pharmacopoeia Volume I \& II, Monographs: Medicinal and Pharmaceutical Substances, Ph. Eur. monograph 0386.

10. M.N.G. James, G.J.B. Williams, Can. J. Chem., 1974, 52, 1872-1879.

11. C. Bertucci, D. Tedesco, J. Chromatogr. A 1269, 69-81, (2012)

12. P.D. Rice, Y.Y. Shao, S.R. Erskine, T.G. Teague, D.R. Bobbitt, Talanta 36, 473-478, (1989)
13. S. Sinnecker, A. Rajendran, A. Klamt, M. Diedenhofen, F. Neese, $J$ Phys. Chem. A. 110, 2235-2245, (2006)

14. T. Bruhn, A. Schaumlöffel, Y. Hemberger, G. Bringmann, SpecDis version 1.62, University of Wuerzburg, Germany, 2014. (http://wwworganik.chemie.uni-wuerzburg.de/lehrstuehlearbeitskreise/bringmann/ specdis/)

15. F. Neese, WIREs Comput. Mol. Sci. 2, 73-78, (2012)

16. M. Valiev, E.J. Bylaska, N. Govind, K. Kowalski, T.P. Straatsma H.J.J. van Dam, D. Wang, J. Nieplocha, E. Apra, T.L. Windus, W.A de Jong, Comput. Phys. Commun. 181, 1477-1489, (2010)

17. Lux Columns Complete Chiral Solutions Catalogue, Phenomenex, Torrance, CA, USA (2013).

18. P.J. Stephens, F.J. Devlin, J.R. Cheeseman, M.J. Frisch, Chirality, 2002, 14, 288-296.

19. J. Autschbach, S. Patchkovskii, T. Ziegler, S.J.A. van Gisbergen, E.J. Baerends, J. Chem. Phys., 2002, 117, 581-592.

20. P.J. Stephens, J.-J. Pan, F.J. Devlin, M. Urbanová, J. Hájíček, J. Org. Chem., 2007, 72, 2508-2524.

21. M. Kwit, J. Gawronski, L. Sbircea, N.D. Sharma, M. Kaik, D.R. Boyd, Chirality, 2009, 21, E37-E47.

22. P.L. Polavarapu, N. Jeirath, T. Kurtán, G. Pescitelli, K. Krohn, Chirality, 2009, 21, E202-E207.

23. P.J. Stephens, J.J. Pan, F.J. Devlin, J.R. Cheeseman, J. Nat. Prod., 2008, 71, 285-288

24. M.A. Muñoz, M.A. Bucio, P. Joseph-Nathan, Nat. Prod. Commun., 2013, 8, 1075-1078.

25. E. Giorgio, N. Parrinello, S. Caccamese, C. Rosini, Org. Biomol. Chem., 2004, 2, 3602-3607. 